\title{
Crónica de la jurisprudencia del Tribunal Constitucional sobre igualdad (año 2020)
}

\author{
Chronicle of the Spanish Constitutional Court \\ case on equality rights (year 2020)
}

\author{
Ricardo Pedro Ron Latas \\ Profesor Titular de Universidad de Derecho del Trabajo \\ y Seguridad Social. Universidade da Coruña \\ Magistrado Suplente. Sala de lo Social del TSJ Galicia
}

doi: https://doi.org/10.20318/femeris.2021.6145

Resumen. Este estudio aborda el análisis de las sentencias dictadas en aplicación del principio de igualdad y no discriminación por el Tribunal Constitucional el año 2020. El estudio se centra en el estudio de las sentencias más relevantes en este terreno que ha producido el Tribunal Constitucional en el año 2020.

Palabras clave: Tribunal Constitucional; principio de igualdad de trato; no discriminación.

Abstract. This study tackle the analysis of judgments dictated in application of the principle of equal treatment and nondiscrimination by the Constitutional Court of Justice of Spain during the year 2020. The article will focus in the study of the most notable sentences in this terrain that has produced the Constitutional Court during the year 2020.

Keywords: Constitutional Court of Justice of Spain; principle of equal treatment; nondiscrimination.

El año 2020, el año de la pandemia, en el ámbito de la jurisprudencia dictada por el Tribunal Constitucional en aplicación del principio de igualdad de trato y no discriminación que consagra el artículo 14 de la $\mathrm{CE}$, pueden reseñarse dos resoluciones significativas, esto es, dos menos que al año pasado.

\section{Sentencia 79/2020 de 2 de julio. Reducción de jornada por guarda legal. Difer- encia de trato de los trabajadores a tiempo parcial respecto de los trabajadores a tiempo completo.}

La primera de esas dos sentencias resulta ser la número 79/2020, de 2 de julio, dictada por el pleno del Tribunal Constitucional. En ella se tratan dos temas ya clásicos 
en la jurisprudencia constitucional: la discriminación indirecta por razón de género y el derecho a la igualdad, ambos (como es bien sabido) reconocidos en el art. 14 CE. El supuesto de hecho era relativamente sencillo. Se trataba de una médica del servicio de urgencias del Hospital Costa del Sol de Marbella (Málaga), que venía realizando una jornada ordinaria de 1.523 horas de trabajo (a razón de 7 horas al día) y otra complementaria como consecuencia de la realización obligatoria de 44 guardias al año (en horario nocturno, con una duración de 10 horas), cada una de las cuales, no solo generaba al día siguiente ("saliente de guardia") un descanso de veinticuatro horas, sino que, a efectos retributivos, se tomaban 7 de esas horas como de trabajo efectivo (descanso retribuido). De este modo, en el cómputo anual de las 1.523 horas de jornada ordinaria de la trabajadora quedaban incluidas las 308 horas generadas por los "salientes de guardia". Posteriormente, solicitó la aplicación de la reducción de jornada por motivos de guarda legal para el cuidado de los hijos menores de doce años (en un 33 por ciento) prevista en el art. 37.6 del Estatuto de los Trabajadores. En consecuencia, la empresa procedió a reducirle, en la misma proporción, el número de horas de su jornada ordinaria (que pasó de 1.523 a 1.020,41 horas), el número de guardias médicas obligatorias (que pasó de 44 a 30), así como el número de horas de descanso retribuido computables por cada saliente de guardia (reduciéndolo de 7 a 4,69 horas).

La trabajadora, sin embargo, entendió que la decisión empresarial no se ajustaba a Derecho. Y es que, para ella la reducción aplicada sobre el descanso retribuido por guardia médica resultaría contraria al principio de igualdad y no discriminación del art. $14 \mathrm{CE}$, ya que producía una diferencia de trato injustificada entre trabajadores a tiempo completo y trabajadoras con jornada reducida, de manera que, a igualdad de jornada (guardia médica obligatoria de 10 horas), recibía una menor compensación por cada "saliente de guardia" (de 4,69 horas en lugar de 7 horas), de modo que tendría que trabajar 69,30 horas más en términos anuales (un total de 879,71 horas en lugar de 810,41 horas). Además, podría producirse también una discriminación indirecta por razón de sexo, en la medida en que el trato desigual denunciado vendría motivado por el ejercicio de un derecho (el de reducción de jornada para el cuidado de hijos) que, aunque reconocido legalmente tanto a los hombres como a mujeres, se ejercita mayoritariamente por estas últimas.

Y esas dos fueron justamente las cuestiones que se resolvieron por el Tribunal Constitucional. La primera de ellas, la relativa a la vulneración del principio de igualdad de trato, exigía, obviamente, un pequeño repaso a la ya añeja doctrina constitucional sobre el derecho a la igualdad y no discriminación. Y así se hizo, recordando la sentencia 79/2020 que "el derecho a la igualdad reconocido en el primer inciso del art. $14 \mathrm{CE}$ exige que a iguales supuestos de hecho se apliquen iguales consecuencias jurídicas, lo que veda la utilización de elementos de diferenciación que quepa calificar de arbitrarios o carentes de una justificación objetiva y razonable". El juicio de igualdad, pues, "siendo relacional, exige como presupuestos obligados, de un lado, que se haya introducido directa o indirectamente una diferencia de trato entre grupos o categorías de personas; de otro, que las situaciones subjetivas que quieran traerse a la comparación sean, efectivamente, homogéneas o equiparables, es decir, que el término de comparación no resulte arbitrario o caprichoso. 
Sólo una vez verificado uno y otro presupuesto resulta procedente entrar a determinar la licitud constitucional o no de la diferencia de trato".

Sobre esta base, la resolución que nos ocupa determinó que, a la vista de lo acreditado en pleito, y teniendo en cuenta la síntesis doctrinal anterior, existía una evidente diferencia de trato contraria al art. $14 \mathrm{CE}$. La razón no era otra que la necesaria consecuencia derivada del hecho de que la empleadora, al calcular la reducción de jornada por motivos de guarda legal para el cuidado de los hijos menores, no solo aplicó la reducción al número de horas de la jornada ordinaria (que pasó de 1.523 a 1.020,41 horas, en jornadas diarias de 7 horas) y al número de guardias médicas obligatorias (que pasó de 44 a 30, en jornadas diarias de 10 horas), sino también al número de horas de descanso retribuido computables por cada saliente de guardia, reduciéndolo de 7 horas a 4,69 horas. Y siendo así, si el juicio de igualdad es de carácter relacional, requiriendo que la diferencia de trato se produzca entre grupos o categorías de personas que puedan considerarse homogéneas o equiparables, resulta que en esta ocasión las situaciones subjetivas que se concretaron en el pleito constituyen un término de comparación válido, al tratarse de supuestos de hecho iguales en la medida que, aun realizando menos guardias obligatorias (30 en lugar de 44, fruto de la reducción de jornada para el cuidado de los hijos), las que realiza la trabajadora recurrente en amparo son, al igual que el resto de sus compañeros, de 10 horas de duración.

Estando, pues, ante situaciones subjetivas comparables, que son aptas para realizar el juicio de igualdad, resultó que la diferencia de trato denunciada no poseía una justificación objetiva y razonable que la legitimase. El argumento que ofrecía la empresa para justificar la diferencia de trato (y que había sido aceptado en la vía judicial) es que, dado que la trabajadora tiene su jornada habitual reducida en un 33 por ciento, las guardias realizadas no pueden generarle un descanso retribuido equivalente a una jornada de trabajo "ordinaria" completa (de 7 horas), procediendo a reducírselo a 4,69 horas. Para el Tribunal Constitucional, sin embargo, tal argumentación resultaba inasumible, al utilizarse, para justificar la diferencia de trato, un elemento de diferenciación que debe calificarse de arbitrario o carente de una justificación objetiva y razonable, esto es, el de que la recurrente en amparo realiza la jornada de trabajo (tanto ordinaria como complementaria) de forma reducida.

No hay que descuidar, se afirma en la sentencia, "que la reducción tanto de la jornada ordinaria (en cómputo anual) como de la complementaria (en número de guardias) es fruto de un derecho (el cuidado de los hijos), cuyo ejercicio no puede generarle un menoscabo en la asignación del descanso retribuido derivado de la realización de unas guardias que, aun menores en su número, son de la misma duración que para el resto de los trabajadores: de 10 horas". Dicho de otra manera, como se ha señalado ya, aunque la recurrente realiza menos jornadas "ordinarias" de trabajo (1.020,41 horas anuales en lugar de las 1.523 horas previstas, en jornadas diarias de 7 horas), y menos jornadas "complementarias" (30 guardias obligatorias en lugar de las 44 previstas, en jornadas de 10 horas), fruto de la reducción para el cuidado de los hijos, las que sí realiza (las 30 guardias), las lleva a cabo, al igual que el resto de sus compañeros, a jornada completa de 10 horas. Por tanto, ante una misma situación (guardias de 10 horas), que generan el derecho a un descanso 
retribuido (de 7 horas), no puede la empleadora asignarle otro (de 4,69 horas), so pretexto de que ya tiene reducido tanto el número de horas ordinarias que debe trabajar como el número de guardias que obligatoriamente debe realizar. No existe, pues, una justificación razonable que legitime el diferente trato recibido.

En segundo lugar, la sentencia procedió a determinar si con la actuación empresarial se había producido una discriminación por razón de género. En estos casos, lo primero es recordar que la virtualidad del art. 14 CE no se agota en la proclamación del derecho a la igualdad, sino que el precepto se refiere también a la prohibición de discriminación, entre otros motivos, "por razón de sexo". Y como se sabe tal tipo de discriminación comprende no solo el tratamiento peyorativo "que se funda en la pura y simple constatación del sexo de la persona afectada, sino en la concurrencia de razones o circunstancias que tengan con el sexo de la persona una conexión directa e inequívoca, como sucede con el embarazo y la maternidad". No obstante, esta protección constitucional vinculada a factores diferenciales que inciden en las mujeres no se detiene en el embarazo y subsiguiente maternidad, sino que se extiende también al ejercicio por la trabajadora de los "derechos asociados a la maternidad". Tales derechos, aunque son reconocidos por el ordenamiento jurídico indistintamente al hombre y a la mujer con el objeto de estimular cambios en la cultura familiar y promover el reparto de responsabilidades, "sirven principalmente para compensar las dificultades y desventajas que agravan la posición de la mujer trabajadora. En efecto, se trata de derechos que inciden de modo singular en las mujeres, al ser las que principalmente asumen el cuidado de los hijos de corta edad y sufren, por ello, mayores dificultades para su inserción laboral y su mantenimiento en el mercado de trabajo".

En todo caso, el Tribunal Constitucional también se encarga de subrayar que la discriminación no solo se produce cuando a la mujer trabajadora se le niega uno de esos derechos asociados a la maternidad, sino también cuando, pese a su inicial reconocimiento y ejercicio, se le cause un perjuicio efectivo y constatable que consista en un "trato peyorativo en sus condiciones de trabajo", o en "una limitación o quebranto de sus derechos o legítimas expectativas económicas o profesionales en la relación laboral”. En otras palabras, en tales casos la vulneración del art. 14 CE vendría determinada por el menoscabo, de carácter discriminatorio, ocasionado en el patrimonio jurídico de la mujer trabajadora. $\mathrm{Y}$, entre esos derechos asociados a la maternidad que, como se ha dicho, responden a la idea de compensar las desventajas reales que para la conservación de su empleo y para la conciliación de su vida laboral y familiar soporta la mujer trabajadora, se encuentran, precisamente, el derecho a la excedencia laboral por cuidado de hijos (art. 46.3 del Estatuto de los Trabajadores) y el derecho a la reducción de jornada por razones de guarda legal (art. 37.6 del Estatuto de los Trabajadores).

En fin, recuerda por último el tribunal que también que la específica prohibición de discriminación por razón de género no sólo comprende la "discriminación directa", sino también la "discriminación indirecta", es decir, aquel "tratamiento formalmente neutro o no discriminatorio del que se deriva, por las diversas condiciones fácticas que se dan entre trabajadores de uno y otro sexo, un impacto adverso sobre los miembros de un determinado sexo". 
Para finalizar, la respuesta del Tribunal Constitucional, acerca de si podía apreciarse una discriminación indirecta por razón de género, resultó especialmente rácana. Aunque se admite que sí, que, en efecto, de la decisión empresarial resulta una discriminación indirecta por razón de género, ello se hace mediante una fundamentación ciertamente escasa y carente de justificación objetiva. Para la resolución el método de cálculo usado por la empresa para asignarle a la trabajadora los períodos de descanso retribuidos por cada "saliente de guardia", le ha provocado un "perjuicio efectivo y constatable" que ha generado, en este caso, un "trato peyorativo en sus condiciones de trabajo" fruto del ejercicio de un derecho asociado con la maternidad (como es el derecho a la reducción de jornada para el cuidado de hijos). Hasta aquí todo correcto, el problema es que más adelante se justifica la apreciación de discriminación en que "aunque aquel método es formalmente neutro, ha perjudicado a un número mayor de mujeres que de hombres (STC 91/2019), pues esta demanda de amparo forma parte de una serie de recursos en que todas las recurrentes, a fecha del dictado de esta sentencia, son mujeres médicos", por lo que "al carecer la diferencia de trato de una justificación que la legitime, procede estimar la vulneración del derecho a la igualdad, sin discriminación por razón de maternidad, reconocido en el art. 14 CE”. Es decir, que el Tribunal Constitucional utiliza un concepto, no amplio, amplísimo, en la aplicación de la doctrina judicial.

Como se sabe, en estos casos el Tribunal de Justicia de la Unión Europea viene concluyendo que la existencia de la desventaja que exige la apreciación de una discriminación indirecta por razón de género "podría quedar demostrada, entre otras formas, probando que una normativa como la controvertida en el litigio principal afecta negativamente a una proporción de personas de un sexo significativamente más alta que la de las personas del otro sexo" (STJUE de 8 de mayo de 2019, Caso V.V.L. contra Instituto Nacional de la Seguridad Social), y siempre "que el juez nacional disponga de datos estadísticos, el Tribunal de Justicia ha declarado que el mejor método de comparación consiste en confrontar, por un lado, las proporciones respectivas de trabajadores que quedan y que no quedan afectados por la norma en cuestión dentro de la mano de obra masculina y, por otro lado, las mismas proporciones dentro de la mano de obra femenina. No basta con considerar el número de personas afectadas, ya que dicho número depende del número de trabajadores activos en todo el Estado miembro, así como de la proporción de trabajadores masculinos y de trabajadores femeninos en dicho Estado miembro". No obstante, eso ha sido justo lo que ha hecho el Tribunal Constitucional, llegando a la conclusión de que en esta ocasión se perjudica a un número mayor de mujeres que de hombres por el simple hecho de que "esta demanda de amparo forma parte de una serie de recursos en que todas las recurrentes, a fecha del dictado de esta sentencia, son mujeres médicos".

Parece, en fin, que el hecho de que en el año 2020 se hayan dictado poco más de media docena de sentencias similares a la que nos ocupa (168/2020 de 16 noviembre, $129 / 2020$ de 21 septiembre, 128/2020, de 21 de septiembre, 124/2020, de 21 de septiembre, 120/2020, de 21 de septiembre, 90/2020, de 20 julio y 91/2020, de 20 de julio), justifica para el Tribunal Constitucional la constatación de una situación discriminatoria, seguramente mediante la utilización del criterio sentado por el Tribunal Europeo según el 
cual "corresponde al órgano jurisdiccional nacional apreciar en qué medida los datos estadísticos presentados ante él, que representan la situación de la mano de obra, son válidos y si se pueden tomar en consideración, es decir, si no constituyen la expresión de fenómenos meramente fortuitos o coyunturales y si, de manera general, resultan significativos".

\section{71/2020 De 29 junio. Discriminación por razón de género. Licencias por hospi- talización tras dar a luz. La novedosa "discriminación refleja"}

La segunda de las resoluciones anunciadas resulta ser la sentencia número 71/2020, de 29 de junio, que se ocupa de nuevo de la discriminación por razón de género, tanto directa como indirecta. En esta ocasión se trataba de una enfermera del Servicio Vasco de Salud-Osakidetza, que solicitó la concesión de dos días de licencia por hospitalización de su hermana por razón de parto, invocando a tal efecto el art. 47 del Decreto 235/2007, de 18 de diciembre, por el que se aprueba el acuerdo regulador de las condiciones de trabajo en el Servicio Vasco de Salud, que regula la "licencia por enfermedad grave, hospitalización o fallecimiento de parientes", y donde se establece el derecho a dos días de licencia retribuida "en casos de hospitalización o enfermedad grave" de familiar dentro del segundo grado de consanguinidad (y dos días hábiles más, si los hechos que lo motivan se producen a más de 150 kilómetros del lugar de residencia del solicitante), exigiendo como único requisito el de aportar justificante de hospitalización del familiar, que fue lo que hizo la trabajadora. La empresa, sin embargo, denegó la licencia, por entender que el parto no está incluido en las previsiones de la norma, por lo que tendría que acreditar la existencia de causa para la hospitalización o la concurrencia de enfermedad grave.

A la vista de lo anterior, en la demanda de amparo se sostenía que la denegación infringe el derecho a la igualdad de trato y no discriminación por razón de género (art. 14 $\mathrm{CE}$ ), tanto de forma directa como indirecta. De forma directa porque el alumbramiento se produce únicamente por mujeres a quienes se les deniega la protección y cuidado de sus familiares al impedirles a estos últimos el acceso a la licencia destinada a procurar su cuidado y atención durante la hospitalización, y que está prevista para tal fin en el art. 47.1 del acuerdo regulador de las condiciones de trabajo. Y de forma indirecta por cuanto que, si el familiar por quien el trabajador o trabajadora del Servicio Vasco de Salud solicita el permiso de hospitalización es una mujer, la dirección del centro correspondiente pregunta por el motivo del ingreso hospitalario, mientras que si la misma licencia se solicita por hospitalización de un familiar hombre la dirección no pregunta por el motivo, bastándole el certificado de hospitalización.

Así delimitado el objeto del pleito, el Tribunal Constitucional comienza (como no podía ser de otro modo) sintetizando la doctrina constitucional sobre el principio de igualdad y la prohibición de discriminación (art. 14 CE), y en concreto sobre la discriminación por razón de género y las distintas manifestaciones de dicha discriminación reconocidas por dicha jurisprudencia.En este sentido, el Tribunal ha declarado, en relación con la cláusula de no discriminación por razón de género del art. 14 CE que, “a diferencia del princi- 
pio genérico de igualdad, que no postula ni como fin ni como medio la paridad y sólo exige la razonabilidad de la diferencia normativa de trato, las prohibiciones de discriminación contenidas en el art. 14 CE implican un juicio de irrazonabilidad de la diferenciación establecida ex constitutione, que imponen como fin y generalmente como medio la parificación, de manera que sólo pueden ser utilizadas excepcionalmente por el legislador como criterio de diferenciación jurídica, lo que implica la necesidad de usar en el juicio de legitimidad constitucional un canon mucho más estricto, así como un mayor rigor respecto a las exigencias materiales de proporcionalidad". Recuerda, a su vez, que "la expresa exclusión de la discriminación por razón de sexo contenida en el art. 14 CE responde a la determinación del constituyente de terminar con la histórica situación de inferioridad en que, en la vida social y jurídica, se había colocado a la mitad de la población. Pese a ello, a día de hoy las mujeres aún soportan situaciones de desigualdad y dificultades específicas que se traducen, entre otras consecuencias, en una menor incorporación de la mujer al trabajo o en una mayor dificultad para conciliar la vida personal, familiar y laboral, particularmente por razón de la maternidad". Ante esta realidad el Tribunal ha declarado "en su reciente STC 108/2019, FJ 3, que es necesario — tal y como reclama la sociedad y se refleja en la intervenciones del legislador - abundar en esa protección, ampliar y desplegar su sentido profundo ligado a la dignidad de la persona y valor de todo ser, y tutelar y favorecer el cambio de conciencia y convivencia que solo la paridad garantiza, fortaleciendo la tutela siempre que se constate (como señalara el Pleno de este Tribunal hace escasas fechas en la STC 91/2019, de 3 de julio, FJ 10) una desigualdad histórica que pueda calificarse de 'estructural', pues la igualdad sustantiva es un elemento definidor de la noción de ciudadanía en nuestro orden constitucional (STC 12/2008, de 29 de enero, FJ 4). En definitiva, como señala la STC 66/2014, de 5 de mayo, FJ 2, la prohibición constitucional específica de los actos discriminatorios por razón de sexo determina que se habrá producido la lesión directa del art. $14 \mathrm{CE}$ cuando se acredite que el factor prohibido representó el fundamento de una minusvaloración o de un perjuicio laboral, no teniendo valor legitimador en esos casos la concurrencia de otros motivos que hubieran podido justificar la medida al margen del resultado discriminatorio'".

Por lo que se refiere a las distintas formas de discriminación por razón de sexo que ha abordado la doctrina constitucional, estas se sintetizan en los siguientes términos: "Desde la STC 145/1991, de 1 de julio, este Tribunal incluye en la prohibición de discriminación del art. 14 CE tanto la discriminación directa como la discriminación indirecta. La primera consiste en un tratamiento perjudicial en razón del sexo en el que el sexo es objeto de consideración directa. Y respecto a la discriminación indirecta por razón de sexo, la doctrina constitucional ha asumido el concepto elaborado por la jurisprudencia del Tribunal de Justicia de la Unión Europea, para referirse a aquellas medidas que, aunque formuladas de manera neutra, perjudican a un porcentaje muy superior de mujeres que de hombres: tales medidas están prohibidas salvo que estén justificadas por factores objetivos ajenos a cualquier discriminación por razón de sexo ... En la actualidad ambos conceptos se encuentran también expresamente recogidos tanto en el Derecho de la Unión Europea [art. 2.1) de la Directiva 2006/54/CE , del Parlamento Europeo y del Consejo, de 5 de julio de 2006, 
relativa a la aplicación del principio de igualdad de oportunidades e igualdad de trato entre hombres y mujeres en asuntos de empleo y ocupación] como en el ordenamiento interno (art. 6 de la Ley Orgánica 3/2007, de 22 de marzo, para la igualdad efectiva de mujeres y hombres, ley que incorpora el acervo comunitario sobre igualdad de sexos). La discriminación directa se define en el apartado primero del art. 6 de la Ley Orgánica 3/2007 como "la situación en que se encuentra una persona que sea, haya sido o pudiera ser tratada, en atención a su sexo, de manera menos favorable que otra en situación comparable". Y el art. 8 de la misma Ley especifica que "constituye discriminación directa por razón de sexo todo trato desfavorable a las mujeres relacionado con el embarazo o la maternidad". Mientras que el apartado 2 del art. 6 define la discriminación indirecta como "la situación en que una disposición, criterio o práctica aparentemente neutros pone a personas de un sexo en desventaja particular con respecto a personas del otro, salvo que dicha disposición, criterio o práctica puedan justificarse objetivamente en atención a una finalidad legítima y que los medios para alcanzar dicha finalidad sean necesarios y adecuados".

Con relación a la discriminación directa, el Tribunal ha declarado de forma reiterada y constante, que "comprende no solo aquellos tratamientos peyorativos que encuentren su fundamento en la pura y simple constatación del sexo de la persona perjudicada, sino también los que se funden en la concurrencia de condiciones que tengan con el sexo de la persona una relación de conexión directa e inequívoca (por todas, y por referirnos a las última que lo afirma en esos estrictos términos, STC 2/2017, de 16 de enero, FJ 5), vinculando particularmente con esto último el embarazo y su incidencia en las condiciones de trabajo de la mujer, toda vez que se trata de un elemento o factor diferencial que, en tanto que hecho biológico incontrovertible, incide de forma exclusiva sobre las mujeres. En este sentido, se afirma que 'la protección de la condición biológica y de la salud de la mujer trabajadora ha de ser compatible con la conservación de sus derechos profesionales, de suerte que la minusvaloración o el perjuicio causado por el embarazo o la sucesiva maternidad constituyen un supuesto de discriminación directa por razón de sexo' (en el mismo sentido, por ejemplo, STC 162/2016, de 3 de octubre, FJ 6)".

Por lo que se refiere a la discriminación indirecta, "cuando se denuncia una discriminación indirecta por razón de sexo, no se exige aportar como término de comparación la existencia de un trato más beneficioso atribuido única y exclusivamente a los varones, sino que exista, en primer lugar, una norma o una interpretación o aplicación de la misma que produzca efectos desfavorables para un grupo formado mayoritariamente, aunque no necesariamente de forma exclusiva, por mujeres. Y, en segundo lugar, se requiere que los poderes públicos no puedan probar que la norma que dispensa una diferencia de trato responde a una medida de política social, justificada por razones objetivas y ajenas a toda discriminación por razón de sexo. En suma, como afirmamos en la STC 253/2004, 'en estos supuestos, para que quepa considerar vulnerado el derecho y mandato antidiscriminatorio consagrado en el art. $14 \mathrm{CE}$ debe producirse un tratamiento distinto y perjudicial de un grupo socialformado de forma claramente mayoritaria por mujeres, respecto de bienes relevantes y sin que exista justificación constitucional suficiente que pueda ser contemplada como posible límite al referido derecho (FJ 7)'. La incorporación de la dis- 
criminación indirecta como contenido proscrito por el art. $14 \mathrm{CE}$ repercute en la forma de abordar el análisis de este tipo de discriminaciones, pues deberá atenderse necesariamente a los datos revelados por la estadística (STC 128/1987, de 14[sic] de julio, FJ 6), ya sean datos estadísticos aportados por el órgano judicial promotor de la respectiva cuestión de inconstitucionalidad o datos estadísticos actualizados de oficio por el Tribunal (STC 253/2004, FJ 8)".

Atendiendo a esa amplia y enjundiosa doctrina, el Tribunal Constitucional concluyó que la decisión empresarial vulneró el derecho de la trabajadora a no padecer discriminación por razón de género. La demandante alegaba que las resoluciones del Servicio Vasco de Salud vulneraban el derecho a la igualdad amparado por el art. 14 CE por incurrir tanto en discriminación directa como indirecta por razón de sexo. Argumentaba, en concreto, que incurre en la primera forma de discriminación al introducir un criterio diferenciador que afecta únicamente a las mujeres, a quienes se les deniega la protección y cuidado de sus familiares al impedirles a estos últimos el acceso a la licencia destinada a procurar su cuidado y atención durante la hospitalización prevista en el art. 47.1 c) del acuerdo regulador de las condiciones de trabajo. Consideraba que también incurre en discriminación indirecta porque cuando el familiar por quien el trabajador o trabajadora del Servicio Vasco de Salud solicita el permiso de hospitalización es una mujer la dirección del centro correspondiente pregunta por el motivo del ingreso hospitalario, mientras que si la misma licencia se solicita por hospitalización de un familiar hombre basta con el certificado de hospitalización.

En este caso, la administración sanitaria autonómica lleva a cabo una interpretación del art. 47.l c) del acuerdo regulador de las condiciones de trabajo que implica un trato de la mujer hospitalizada por razón de alumbramiento que es diferente del que se otorga al hombre hospitalizado. Trato desigual que se fundamenta en un hecho biológico, el parto, directamente asociado al embarazo y la maternidad y que, por tanto, incide de forma exclusiva sobre las mujeres. Tal interpretación trae causa de los criterios adoptados en la reunión de la comisión paritaria del acuerdo celebrada el día 13 de febrero de 2004, que consideró que "existe hospitalización cuando ésta sea asimilada a la enfermedad grave", y en virtud de los cuales el Servicio Vasco de Salud considera que entre los supuestos que dan derecho a la licencia del art. 47 acuerdo regulador de las condiciones de trabajo no está incluida la hospitalización por parto, salvo que se acredite que a raíz del mismo concurre otra causa para la hospitalización o una enfermedad grave. Ello pese a que el art. 47.1 c) del acuerdo regulador de las condiciones de trabajo recoge como dos supuestos diferenciados por los que solicitar la licencia, por una parte, la enfermedad grave y, por otra, la hospitalización, sin distinguir entre las causas que motivan esta última ni condicionar el disfrute de la licencia a la concurrencia de otro requisito. De modo que del tenor literal de la misma no se infiere que quepa interpretar que la hospitalización ha de serlo por enfermedad, y que se excluye la hospitalización por parto, por no ser el parto una enfermedad.

Su aplicación en estos términos, supone una diferenciación que implica un trato menos favorable tanto para las mujeres hospitalizadas como para los empleados o empleadas del Servicio Vasco de Salud que solicitan la licencia por hospitalización de mujeres de su familia. A diferencia de lo que ocurre cuando se solicita la licencia por hospitalización 
de un hombre, cuando un empleado o empleada solicita la licencia por hospitalización de una mujer la Administración requiere que se justifique no solo el ingreso hospitalario del familiar, sino también el motivo de dicho ingreso a fin de determinar si se trata de un caso de hospitalización por alumbramiento en el que no concurra enfermedad grave de la mujer, y denegar la licencia en estos casos. De modo que, por una parte, se priva en estos supuestos a los empleados y empleadas del Servicio de Salud Vasco de una licencia a la que tiene derecho conforme al acuerdo regulador vigente. Por otra, a las mujeres hospitalizadas por esta razón se les priva de que sus familiares puedan facilitarles asistencia durante la hospitalización por parto, con el apoyo de esta medida.

En este caso, sin embargo, el tribunal entiende que la lesión del art. 14 CE por discriminación por razón de género no encaja fácilmente en las categorías de discriminación directa o indirecta. Básicamente por lo siguiente: 1) comenzando por la discriminación indirecta, la interpretación y aplicación del art. 47.1 c) del acuerdo regulador de las condiciones de trabajo no puede categorizarse como una discriminación indirecta a la luz de la doctrina constitucional, ya que en este caso no se está ante una interpretación o criterio formulado de manera neutra ni aparentemente neutra, pero que perjudique a un porcentaje muy superior de mujeres que de hombres, antes al contrario, excluye abiertamente la aplicación de la medida a los supuestos de hospitalización por razón de parto cuando, obviamente, solo las mujeres son hospitalizadas por esta razón para recibir la asistencia médica necesaria, por lo que se trata, en definitiva, de una interpretación que se fundamenta en un motivo directamente relacionado con el embarazo y la maternidad y que, en principio, implica una discriminación directa de la mujer; y 2) la característica o condición que conlleva la discriminación directa por razón de género (el alumbramiento) no se sitúa en la persona que solicita acogerse a la licencia como medida, sino que la discriminación se refiere al familiar hospitalizado.

Estas circunstancias, sin embargo, no impidieron al tribunal concluir que se ha vulnerado el art. $14 \mathrm{CE}$ por incurrir la Administración en una discriminación directa por razón de género. La razón es que "lo determinante en las decisiones de la Administración por las que se denegó la licencia a la recurrente es una interpretación del art. 47.1 c) del acuerdo regulador de las condiciones de trabajo que se funda en un criterio directamente discriminatorio por razón de sexo en relación con los supuestos de hospitalización que dan derecho a dicha licencia (al excluir la hospitalización por alumbramiento y, en definitiva, por causa de maternidad, un valor protegido conforme a nuestra jurisprudencia)". Y es que, "tal discriminación proyecta también consecuencias negativas o perjudiciales, además, sobre las empleadas o empleados del Servicio Vasco de Salud que solicitan la licencia por asistir a su pariente hospitalizada. Se les deniega así, por un motivo discriminatorio prohibido por el art. $14 \mathrm{CE}$, la posibilidad de acogerse a dicha licencia para prestarles su ayuda, menoscabando a su vez sus derechos. En definitiva, dichos familiares sufren las consecuencias de una discriminación por razón de sexo prohibida por el art. 14 CE, de manera refleja, por su vinculación familiar con la mujer que está hospitalizada por parto".

Es lo que el Tribunal Constitución denomina, de forma novedosa, como "discriminación refleja", esto es, "cuando una persona es tratada de forma menos favorable por causa 
de su vinculación o asociación con otra que posee uno de los rasgos o características protegidas (o las causas de discriminación prohibidas), pese a no poseer dicha característica en quien alega el trato discriminatorio". En este sentido se ha pronunciado el Tribunal de Justicia de la Unión Europea, que en su sentencia de 17 de julio de 2008 (asunto C-303/06, Coleman), que ha procedido a una interpretación amplia del alcance de los supuestos protegidos por la Directiva 2000/78/CE del Consejo, de 27 de noviembre de 2000, relativa al establecimiento de un marco general para la igualdad de trato en el empleo y la ocupación, al declarar que se incurre en discriminación directa cuando una persona es tratada de forma menos favorable por razón de uno de los rasgos o características protegidos, aunque no concurran en ella misma, si el motivo del trato menos favorable se fundamenta en dicha característica. En el mencionado asunto Coleman, en el que la Gran Sala aborda en concreto un supuesto de discriminación por razón de discapacidad, se declara que los objetivos y el efecto útil de esta Directiva "se verían comprometidos si un trabajador que se encuentre en una situación como la de la demandante en el litigio principal no pudiera invocar la prohibición de discriminación directa establecida en el artículo 2, apartado 2, letra a), de la misma Directiva cuando se haya probado que ha recibido un trato menos favorable que el que recibe, ha recibido o podría recibir otro trabajador en situación análoga, a causa de la discapacidad de un hijo suyo, y ello aunque el propio trabajador no sea discapacitado". De igual forma, el Tribunal Europeo de Derechos Humanos ha declarado en su sentencia de 22 de marzo de 2016 (asunto Guberina contra Croacia, parágrafo 79) que el trato menos favorable recibido por el recurrente por causa de la discapacidad del hijo a su cuidado es una forma de discriminación por razón de discapacidad, prohibida por el art. 14 del Convenio europeo de derechos humanos, aunque el propio recurrente no sufra de discapacidad alguna.

Y eso es justo lo que sucede en este caso, el factor prohibido (la discriminación por razón de género) constituye el fundamento de la denegación de la licencia solicitada conforme al art. 47.1 c) del acuerdo regulador de las condiciones de trabajo, ocasionándole también un perjuicio laboral a la solicitante, aunque no sea en ella, sino en su hermana, en quien concurra la condición que da lugar a la discriminación (la hospitalización por razón de parto). En esas circunstancias, y para hacer efectiva la prohibición de discriminación por razón de género contenida en el art. $14 \mathrm{CE}$, es preciso una vez más "abundar en esa protección, ampliar y desplegar su sentido profundo ( STC 108/2019, FJ 3) concluyendo, en consecuencia, que la aplicación en estos términos del art. $47.1 \mathrm{c}$ ) del acuerdo tantas veces citado por el Servicio Vasco de Salud en las resoluciones por las que denegó a la recurrente la licencia por hospitalización de su hermana, es contraria a la prohibición constitucional específica de actos discriminatorios por razón de sexo, lesionando a su vez de manera refleja el derecho de la recurrente a la igualdad y a la no discriminación". 